\title{
Manuscript Status
}

\author{
Katrina A. Bramstedt
}

Received: 11 April 2014 / Accepted: 15 April 2014 / Published online: 6 May 2014

C) Springer Science+Business Media Dordrecht 2014

Where, oh where, has my manuscript gone?

Where, oh where, can it be?

With its word count cut short and its review time long

Where, oh where, can it be?

I worked so hard

I worked so long

Off it went

Off it's gone
When will it come back to me?

Days and weeks

Months, a year

When will it re-appear?

I think I see it

No, a mirage

I'm waiting for you, fingers crossed.

K. A. Bramstedt $(\square)$

Bond University School of Medicine, University Drive,

Gold Coast, QLD 4229, Australia

e-mail: txbioethics@yahoo.com 